\title{
Correction of Sleep Staging Based on Sleep Spindle Detection
}

\author{
Xue-mei $\mathrm{JIN}^{1}$, Jun-zhong ZOU ${ }^{2, *}$ and Jian $\mathrm{ZHANG}^{3}$ \\ ${ }^{1,2,3}$ East China University of Science and Technology, Shanghai 200037, China \\ ${ }^{*}$ Corresponding author
}

Keywords: Sleep spindles, Sleep staging, Time-domain condition.

\begin{abstract}
It has been demonstrated that sleep has a great relationship with humans' health and activities. Sleep staging is an important method to analysis sleep. Lots of methods have been adopted for automatic sleep staging. Some of the results are good and some of them are poor. For the poor, the sleep spindle detection can help to correct the results, because in the sleep stage 2(S2), the characteristic wave is sleep spindle and in the later of sleep stage 1(S1), the waves less than the duration time of sleep spindles but have the same period with sleep spindles (immature sleep spindles) appear on the electroenphalography (EEG). Therefore, a time-domain method for detecting sleep spindles is mentioned in this paper. With this method, the majority of sleep spindles and immature sleep spindles can be detected and the duration time of these detected waves can be used as the feature for sleep staging correction, especially the results of S1 and $\mathrm{S} 2$. The experiment results show that the accuracy of sleep staging is increased from $65.68 \%$ to $66.22 \%, 47.2 \%$ to $48 \%$ of S1 and $72.4 \%$ to $74.3 \%$ of S2.
\end{abstract}

\section{Introduction}

Sleep is an important physiological activity of humans. About $1 / 3$ of humans life is spent on the sleep. The quality of sleep affect the health of humans and poor sleep quality can cause diseases, such as cerebrovascular, cardiovascular and hypertension [1]. The study on sleep staging can assess the sleep quality effectively.

Researches have shown that the night sleep is not a single process it has a complex structure with a variety of physiological activities. In 1968, the classical criterion of $\mathrm{R} \& \mathrm{~K}$ for sleep staging was proposed in a expert group meeting organized by Kales and Rechtschaffen [2]. According to the criterion, the night sleep is divided into wake(W), rapid eye movement(REM) and non-rapid eye movement(NREM), and the NREM includes S1, S2, stage 3(S3) and stage 4(S4). In 2007, the latest criterion for sleep staging was introduced by American Academy of sleep medicine (AASM), in which the S3 and S4 are merged into slow waves stage (SS) [3]. In this paper, the night sleep is divided into $\mathrm{W}, \mathrm{S} 1, \mathrm{~S} 2$, SS and REM.

Sleep staging has been realized by artificial demarcation for a long time which usually costs much time and is subjective. Now, lots of methods of auto sleep staging were proposed by researchers. Some results of these methods are poor, especially the accuracy of S1 and S2 staging. For example, the sleep staging method based on the improved $K$ means algorithm proposed by Xiao S [4]. Processed by this method, a total accuracy of sleep stage is $76 \%, 14 \%$ for S1, $68 \%$ for S2, $85 \%$ for SS and $70 \%$ for REM. Shown from the results, the staging results of S1 and S2 are relatively poor. If the staging accuracy of S1 and S2 can be improved, the night sleep staging will be good.

As the characteristic wave of S2, the research of sleep spindles is necessary. Sleep spindles generated from complex interactions between thalamic, limbic and cortical areas are the hallmarks of S2. They are sinusoidal spindle-like waveforms which have 
the characteristic of progressively increasing, then gradually decreasing lasting $0.5-3 \mathrm{~s}$ with a frequency profile at $11-16 \mathrm{~Hz}$ [5]. The figure characteristic of the waveforms is obvious.

In this paper, a time-domain method is adopted to detect the sleep spindles and immature sleep spindles [6]. The duration time of detected waves can be used as the discrimination condition. If the duration time of detected waves is less than $0.5 \mathrm{~s}$, the samples that contain these detected waves are belonged to S1, else they are S2's samples.

What follows covers the introduction of method for detecting sleep spindles, the details of correction for sleep staging results, the analysis of the experiment results and the summary of this paper.

\section{Data for experiment}

In this paper, the experiment data was downloaded from the MIT database [7-8]. Three subjects' night sleep data was taken for sleep staging. The length of the subject 1's night sleep is $8.15 \mathrm{~h}, 6 \mathrm{~h}$ of subject 2 and $7.6 \mathrm{~h}$ of subject. Each of the data was divided into many samples at the length of $30 \mathrm{~s}$, and each of them represents just one sleep stage.

\section{Method of detecting sleep spindles}

The time-domain method to detect sleep spindles depends on the algorithm of the merger of increasing or decreasing sequence based on the visual organization principle which was proposed by Zhang $\mathrm{J}$ [9-10]. Visual organization principle is the method to make the basic image elements form an overall subject according to the quantitative model of Gestalt principle in a narrow sense [11]. The merge result is shown in the figure 1 .

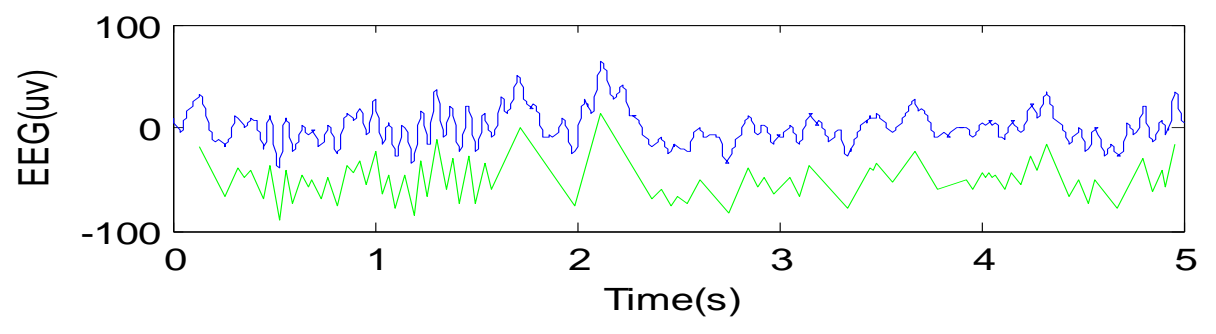

Figure 1. Schematic diagram of EEG signal merging

\section{Algorithm of time-domain method for detecting}

After processed by this merger algorithm, the time-domain feature of EEG signal can be more obvious. The time-domain feature period and duration time are used to detect the sleep spindles. Change the duration time condition, the immature sleep spindles can also be detected. Three steps are taken to detect the sleep spindles.

Step 1: detect the signal wave that satisfied with the period condition of sleep spindle. As shown in figure 2, $T_{1}$ and $T_{2}$ represent period, which are calculated according to the equation (1). 


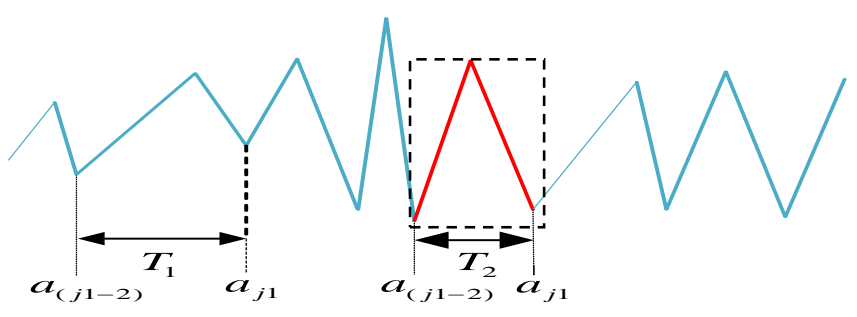

Figure 2. Schematic diagram of the waves satisfied with the periodic condition

$$
T=\left(a_{j 1}-a_{j 1-2}\right) / f_{S}
$$

Shown as the figure 2 , the $a_{j 1}$ represents the location of the merger point in the waveform. In equation (1), $f s$ represents sample frequency. The wave with period in the range of $0.06<T<0.1$ was marked in red in the dashed box.

Step 2: combined the marked waves. After the filter of the period, the single waves meet condition would be marked in red in dashed box. If the interval distance between two adjacent marked waves meets the threshold conditions, the two adjacent marked waves can be combined, which means the waves between two adjacent marked waves would be marked. Shown in the figure $3, L_{1}$ and $L_{2}$ represent the interval distance, which are calculated by equation (2)

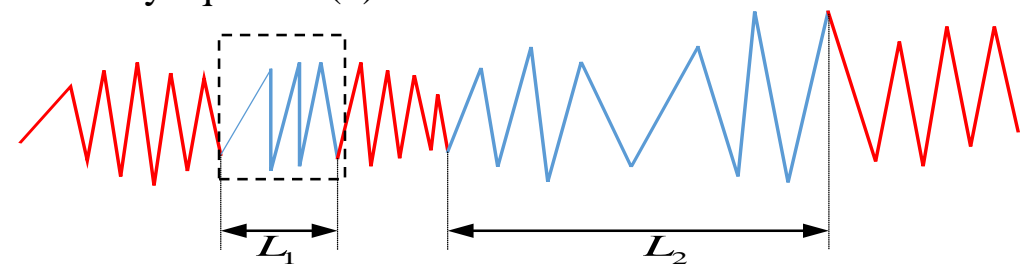

Figure 3. Schematic diagram of the waveform after periodic screening

$$
L=\left(a_{t 1(i+1)}-a_{t(i)}\right) / f s
$$

In the equation (2), $f_{S}$ represents sample frequency. $a_{t \mid(i+1)}$ and $a_{t(i)}$ record the end point and front point of the unmarked waves in the sample waveform. When $L$ meets the condition: $L<\theta$, the waves would be marked shown in the dashed box.

Step 3: using the duration time condition to detect sleep spindles and immuate sleep spindles. Shown in the figure $4, L_{3}$ and $L_{4}$ represent the duration time of marked waves in step 2 , which are calculated by equation (3)

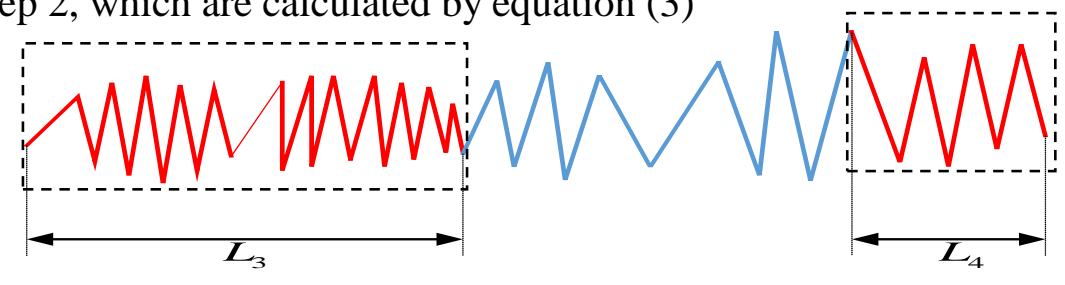

Figure 4. The waves after combined

$$
L=\left(a_{t l(j)}-a_{t L(j)}\right) / f s
$$

In the equation(3), $f_{s}$ represents sample frequency. $a_{t(j)}$ and $a_{t L(j)}$ record the end point and front point of the marked waves in the sample waveform. When $L$ meets the condition: $0.3<L \leq 0.5$, the marked wave is immuature sleep spindle, when $L$ meets 
the condition $0.5<L<3$, the marked wave is sleep spindle. When $L$ meets any other condition, the marked wave is nither the sleep spindle nor the immuature sleep spindles and will be removed the mark. The detected sleep spindle is shown in the figure 5, which was in the dashed box.

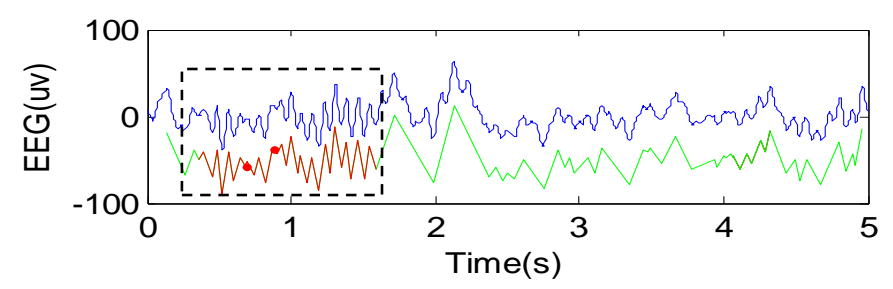

Figure 5. The detected waves

\section{The correction for sleep staging based on the detected waves}

Introduced by the "Textbook of polysomnogramology" written by Tong M [6], the characteristic waves of S1 appearing on the EEG include sharp wave of parietal region, $\theta$ wave, immature sleep spindle and immature k-complex wave and those of S2 are sleep spindle and k-complex wave.

\section{Auto sleep staging results}

In this paper, the auto sleep staging results were gotten by using the sleep staging algorithm based on the improved $K$ means cluster proposed by Xiao S [4]. According to the sleep label, the distributions of each sleep staging in each subject was shown in the table 1 .

Table 1. The distribution of sleep stages

\begin{tabular}{|c|c|c|c|c|c|c|}
\hline Subjects & W & S1 & S2 & SS & REM & total \\
\hline Subject 1 & 62 & 121 & 480 & 96 & 219 & 978 \\
\hline Subject 2 & 68 & 58 & 250 & 220 & 125 & 721 \\
\hline Subject 3 & 60 & 54 & 288 & 298 & 215 & 915 \\
\hline Total & 190 & 233 & 1018 & 614 & 559 & 2614 \\
\hline
\end{tabular}

\section{The correction for the result of sleep staging based on the detected waves}

In the chapter of Time-domain method for detecting algorithm, the range of the duration time feature $L$ was set from $0.3 \mathrm{sec}$ to $3 \mathrm{sec}$ in step 3 . The serial numbers of the samples that have the detected waves were recorded in the array $x_{1}[]$. Each of these samples may have more than one detected wave, the average length $L L$ of these detected waves was taken as the duration time feature of one sample recorded in the array $x_{1}[]$. If $L L$ meets the condition of $0.3<L L \leq 0.5$, the serial numbers in " $x_{1}$ " will be recorded in the array number $S 1[]$, else the serial numbers in the array $x_{1}[]$ will be recorded in the array numberS2[]. The algorithm flow chart is shown as the figure 6 . The sleep staging label achieved by the auto sleep staging algorithm based on the improved $K$ means cluster algorithm [4] was recorded in the array $x_{2}[]$. In " $x_{2}[]$ ", the samples are marked in S1 when the serial numbers of the samples are recorded in numberS1[] and will be marked in S2 when the serial numbers of the samples are recorded in numberS2[]. The accuracy of sleep staging is calculated by the equation (4) and the results before and after the correction are shown in the table 2 . 


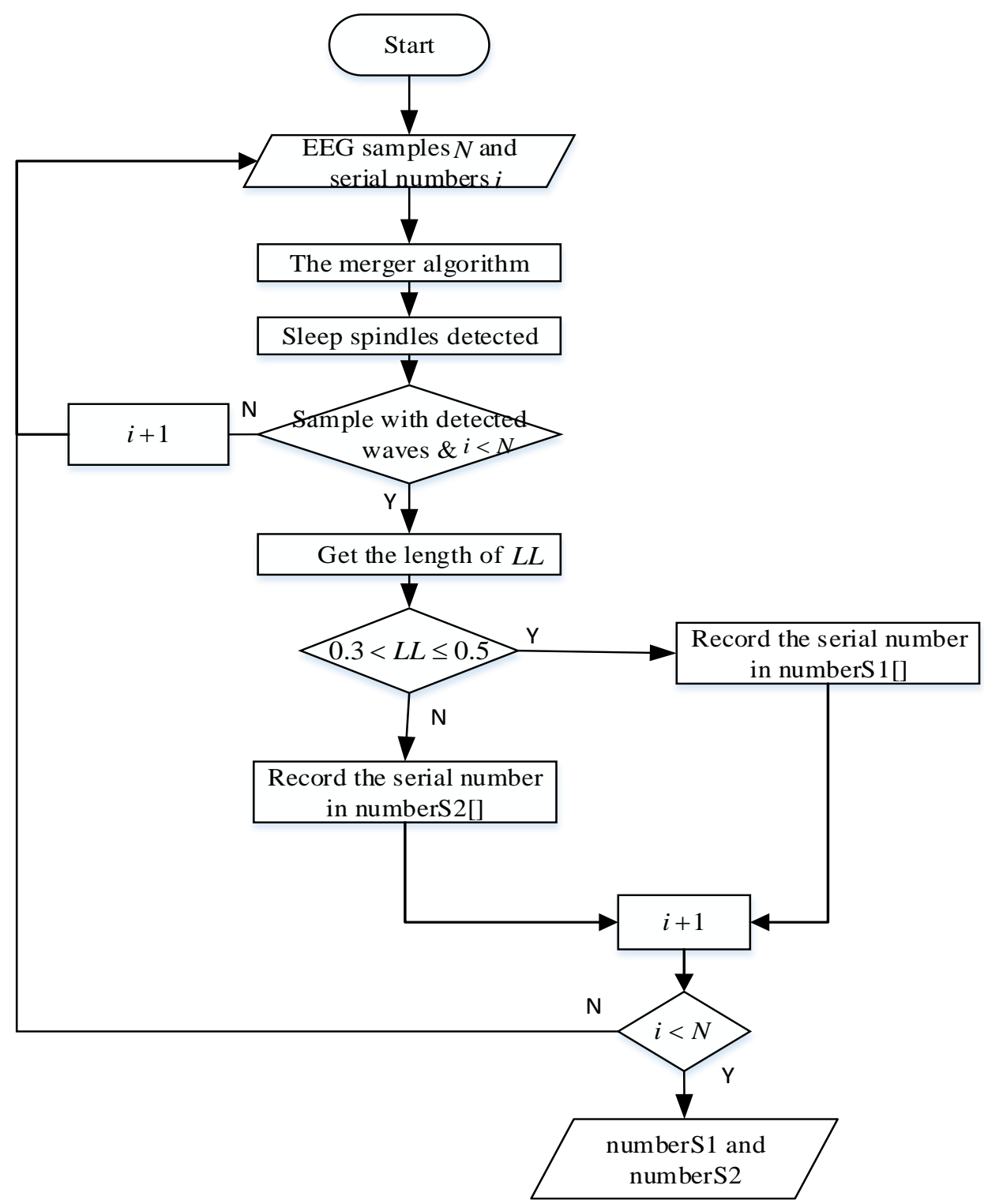

Figure 6. The flow chart for achieving the order number of samples belonged to S2 and S1

Table 2. The accuracy of sleep stage before and after correction

\begin{tabular}{|c|c|c|c|c|c|c|c|}
\hline \multicolumn{2}{|c|}{ subjects } & W & S1 & S2 & SS & REM & total \\
\hline \multirow{2}{*}{$\begin{array}{c}\text { Subject } \\
1\end{array}$} & Before & $53.61 \%$ & $47.11 \%$ & $67.29 \%$ & $93.75 \%$ & $6.39 \%$ & $52.86 \%$ \\
\cline { 2 - 8 } & After & $53.61 \%$ & $48.76 \%$ & $70.42 \%$ & $87.50 \%$ & $6.39 \%$ & $53.89 \%$ \\
\hline \multirow{2}{*}{$\begin{array}{c}\text { Subject } \\
2\end{array}$} & Before & $29.41 \%$ & $55.17 \%$ & $80.00 \%$ & $93.18 \%$ & $82.40 \%$ & $77.67 \%$ \\
\cline { 2 - 8 } & After & $29.41 \%$ & $55.17 \%$ & $80.40 \%$ & $93.18 \%$ & $82.40 \%$ & $77.81 \%$ \\
\hline \multirow{2}{*}{$\begin{array}{c}\text { Subject } \\
3\end{array}$} & Before & $18.33 \%$ & $38.89 \%$ & $74.31 \%$ & $92.28 \%$ & $55.81 \%$ & $70.05 \%$ \\
\cline { 2 - 8 } & After & $18.33 \%$ & $40.74 \%$ & $75.34 \%$ & $91.95 \%$ & $55.81 \%$ & $70.27 \%$ \\
\hline
\end{tabular}

\section{Analysis and Conclusion}

Seen from the table 2, the accuracy of sleep staging can be improved after correction, especially the S1 and S2. The accuracy of S1 has improved in the data of subject 1 and changes without happening in subject 2 and 3. Causes may be as follows: (1) The experiment data was adopted from the channel "Fpz-Cz", in which sleep spindles are less than that adopted from the channel "C4-A1", so the detected waves were few. (2) 
The duration time of Sleep spindle is larger than that of immature sleep spindle, which makes the average duration time $L L$ larger than $0.5 \mathrm{sec}$ and the samples have both sleep spindles and immature sleep spindles are always be marked with S2, so the samples belonged to S1 may be marked with S2. The accuracy of S2 improved a little. When the results of auto sleep staging algorithm are good, most of the samples with sleep spindles will be marked with S2, which may limit the correction of sleep spindles for S2. The accuracy of SS sometimes will decrease, because few sleep spindles appear in the early stage of SS.

Therefore, combine the correction algorithm based on the detection of sleep spindles or any other auto sleep staging algorithm can achieve a great result for sleep staging.

\section{References}

[1] Serhal C E B, Strohl K P. W.J. Randerath, B.M. Sanner, V.K.Somers (eds): Sleep apnea-current diagnosis and treatment (Progress in respiratory research, vol 35)[J]. Sleep and Breathing, 2007, 11(3):205-206.

[2] Brodbeck V, Kuhn A, Wegner F V, et al. EEG microstates of wakefulness and NREM sleep. [J]. Neuroimage, 2012, 62(3):2129-2139.

[3] Xiao M, Yan H, Song J, et al. Sleep stages classification based on heart rate variability and random forest[J]. Biomedical Signal Processing \& Control, 2013, 8(6):624-633.

[4] Xiao S, Wang B, Zhang J, et al. An improved K-means clustering algorithm for sleep stages classification[C]// Conference of the Society of Instrument and Control Engineers of Japan. 2015:1222-1227.

[5] GL De, M Ferrara. Sleep spindles: an overview [J]. Sleep Medicine Reviews, 2003, 7(5): 423-440.

[6] Tong M. The technology and theory of polysomnography [M]. Beijing :People's Military Medical Press, 2004.42-59

[7] Pomerantz J R, Kubovy M. Theoretical Approaches to Perceptual Organization: Si mplicity and Likelihood Principles [M].Handbook of perception and human perfor mance. 1986: 1--45.

[8] Goldberger A L, Amaral L A, Glass L, et al. PhysioBank, PhysioToolkit, and Phy sioNet: components of a new research resource for complex physiologic signals $[\mathrm{J}]$. Circulation, 2000, 101(23): E215.

[9] Zhang J, Zou J, Wang M, et al. Automatic detection of interictal epileptiform disc harges based on time-series sequence merging method [J]. Neurocomputing, 2013, 110(8): 35-43.

[10] Wei Z, Zou J, Zhang J. Automatic recognition of chewing noises in epileptic EEG based on period segmentation [J]. Neurocomputing, 2016, 190: 107-116.

[11] Luo Siwei. Visual perception system and Information processing theory [M]. Beijing: Electronics Industry Press, 2006. 